\title{
Suppression of Charge Equilibration Leading to the Synthesis of Exotic Nuclei
}

\author{
Yoritaka Iwata, ${ }^{1,2}$ Takaharu Otsuka, ${ }^{2,3}$ Joachim A. Maruhn, ${ }^{4}$ and Naoyuki Itagaki ${ }^{2}$ \\ ${ }^{1}$ GSI Helmholtzzentrum für Schwerionenforschung, D-64291 Darmstadt, Germany \\ ${ }^{2}$ Department of Physics, University of Tokyo, Hongo, Tokyo 113-0033, Japan \\ ${ }^{3}$ Center for Nuclear Study, University of Tokyo, Hongo, Tokyo 113-0033, Japan \\ ${ }^{4}$ Institut für Theoretische Physik, Universität Frankfurt, D-60325 Frankfurt, Germany
}

(Received 3 January 2010; published 21 June 2010)

\begin{abstract}
Charge equilibration between two colliding nuclei can take place in the early stage of heavy-ion collisions. A basic mechanism of charge equilibration is presented in terms of the extension of singleparticle motion from one nucleus to the other, from which the upper energy limit of the bombarding energy is introduced for significant charge equilibration. The formula for this limit is presented, and is compared to various experimental data. It is examined also by comparison to three-dimensional timedependent density functional calculations. The suppression of charge equilibration, which appears in collisions at the energies beyond the upper energy limit, gives rise to remarkable effects on the synthesis of exotic nuclei with extreme proton-neutron asymmetry.
\end{abstract}

DOI: 10.1103/PhysRevLett.104.252501

PACS numbers: 21.10.Ft, 25.70.Hi

Charge equilibration is a rapid process during the early stage of heavy-ion collisions with a time scale of $10^{-22} \mathrm{~s}$ (for a review, see [1]). Despite many theoretical attempts, its mechanism has remained an open problem for more than a decade. As far as collective dynamics is concerned, the relation between charge equilibration and the isovector dipole mode has been clarified (for example, see [2-10]). The charge equilibration is quite important, because it naturally prevents the production of exotic fragments with extreme proton-neutron asymmetry. As there are and will be third generation rare-isotope-beam (RI-beam) facilities, it is an urgent question whether such synthesis can be enhanced with higher beam energies or not.

In this Letter, we first point out that there is an upper limit of the bombarding energy for the fast and significant charge equilibration in the initial stage of the collision. This concept is checked using systematic threedimensional time-dependent density functional calculations. Note that the fast charge equilibration appears to occur before dissipation effects become fully operational. We actually employ Skyrme time-dependent Hartree-Fock (TDHF) theory, which is a rather unique presently feasible method for the treatment of nonperturbative processes such as multinucleon transfers in a realistic framework.

The suppression of charge equilibration brings about a favorable situation for the synthesis of exotic nuclei; hence, the evaluation of the upper limit thus can have crucial significance for experiments on nuclei with extreme proton-neutron asymmetry. We shall consider the collision of a target nucleus with mass number $A_{1}$, neutron (proton) number $N_{1}\left(Z_{1}\right)$, with a projectile nucleus with $A_{2}, N_{2}$, and $Z_{2}$. The total mass, neutron, and proton numbers are denoted by $A, N, Z$, respectively. For this problem, we begin with a basic picture that the charge equilibration takes place as wave functions of nucleons propagate from their original nucleus to the other nucleus in the initial stage of the collision. Namely, the regime of individual singleparticle motion spreads out following the lowering of the potential barrier between the two nuclei after the touching. Because this spreading occurs as a consequence of unblocked single-particle motion, the process can be very fast; a particle travels into the other side within $\sim 10^{-22} \mathrm{~s}$, which roughly corresponds to the Fermi energy of normal nuclear matter. Because this spreading needs a certain time, it does not lead to charge equilibration in the initial stage if the relative velocity of the colliding nuclei is too high. In the charge equilibration, protons and neutrons from both nuclei, particularly those near the Fermi levels, are mixed within a short time after the touching.

We introduce an ansatz that, in order for the fast charge equilibration to occur, the relative velocity $v_{r}$ of the two nuclei at the collision must be below the velocities corresponding to the proton or neutron Fermi momenta of both nuclei. By denoting the minimum of these four velocities as $v_{F}{ }^{\min }$, the present effect can be summarized by the statement that the upper-limit energy for charge equilibration is determined by $v_{r}=v_{F}{ }^{\min }$. This upper limit in the laboratory frame for charge equilibration is expressed as the sum of the kinetic energy for velocity $v_{F}{ }^{\text {min }}$ and the Coulomb energy at touching:

$$
\frac{E_{\mathrm{CE}, \mathrm{lab}}}{A}=\frac{\hbar^{2}\left(3 \pi^{2} \rho_{\min }\right)^{2 / 3}}{2 m}+\frac{e^{2} Z_{1} Z_{2}}{4 \pi \epsilon_{0} r_{0}} \frac{A_{1}+A_{2}}{A_{1} A_{2}\left(A_{1}^{1 / 3}+A_{2}^{1 / 3}\right)},
$$

$$
\rho_{\min }=\min _{i}\left(\frac{N_{i}\left(\frac{4 \pi r_{0}}{3} A_{i}^{1 / 3}\right)^{-1}}{(1-3 \bar{\epsilon})(1+\bar{\delta})}, \frac{Z_{i}\left(\frac{4 \pi r_{0}}{3} A_{i}^{1 / 3}\right)^{-1}}{(1-3 \bar{\epsilon})(1-\bar{\delta})}\right)
$$

where $m, e, \epsilon_{0}$, and $r_{0}$ are the nucleon mass, the charge unit, the vacuum permittivity, and the usual nuclear radius parameter $(1.2 \mathrm{fm})$, respectively. Here we express the 


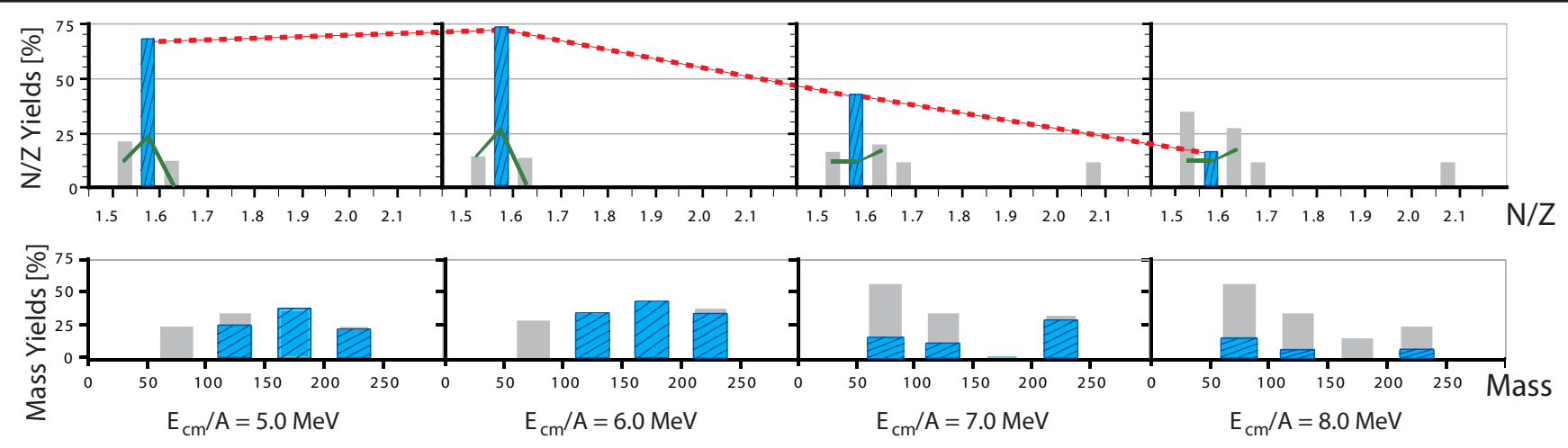

FIG. 1 (color online). Four cases with different $E_{\mathrm{cm}} / A$ values are presented for the collisions of ${ }^{208} \mathrm{~Pb}+{ }^{132} \mathrm{Sn}$. The upper and the lower panels show the yield distributions of fragments obtained by TDHF calculations (SLy4d) as a function of their $N / Z$ ratios and masses, respectively (the $N / Z$ ratio is discretized by 0.05 , and the mass by 50 ), where the $N / Z$ ratios for ${ }^{208} \mathrm{~Pb}$ and ${ }^{132} \mathrm{Sn}$ are 1.54 and 1.64 , respectively. The effective freeze-out time turns out to be $11.5 \times 10^{-22} \mathrm{~s}$. Columns showing the charge equilibrium to the final value $N / Z=1.58$ are hatched, and they are connected by dashed lines for the upper panel. The line graphs shown in the upper panel pick out the peripheral cases with the impact parameter of $7.5 \mathrm{fm}\left(b_{\mathrm{r}}=0.57\right)$.

minimum velocity by the corresponding minimum of the proton or neutron density of the two nuclei using the formula $v_{F}^{\min }=\hbar\left(3 \pi^{2} \rho_{\min }\right)^{1 / 3} / m$. In Eq. (2), $i=1,2$ distinguishes the two initial nuclei, and $\bar{\epsilon}$ and $\bar{\delta}$ (functions of $A_{i}$ ) are introduced based on the droplet model $[11,12]$ to take into account the effect of neutron and proton skins.

Three-dimensional TDHF calculations can give us an insight into the validity of the present conjecture. An estimate of the mean free path of a nucleon in nuclear matter based on the free cross section yields a few femtometers [13]. Since this depends strongly on energy and the phase space blocking through the Pauli principle, it is legitimate to investigate TDHF as an alternative theory at least for the initial phase of a collision and to compare to experiment to find out to what extent hard collisions may influence the results. Many TDHF events were obtained with different values of impact parameter (denoted by $b$ ) incremented by $2.5 \mathrm{fm}$. For easier comparison, in the figure captions, we also mention the impact parameter rescaled according to $b_{\mathrm{r}}=b /\left(r_{0} A_{1}^{1 / 3}+r_{0} A_{2}^{1 / 3}\right)$. These events are summed over impact parameters with weights of geometric cross section, and elastic cases are discarded. Accordingly, the contribution of peripheral collisions is large. Note that nucleon emission is also present in the TDHF calculations. $N / Z$ fragment distributions are obtained by sorting collected TDHF events in terms of the $N / Z$ ratio with $N / Z$ being discretized into bins of width 0.05 . Figure 1 shows the yield distribution of final fragments for ${ }^{208} \mathrm{~Pb}+{ }^{132} \mathrm{Sn}$ reaction. Going from low to high $E_{\mathrm{cm}}$ (total kinetic energy in the center-of-mass frame) of the collision, the peak of the yield distribution as a function of $N / Z$ is almost constant at the beginning, and is shifted later with the lowering of peak height. A clear decrease of the yield of charge-equilibrated fragments for $E_{\mathrm{cm}} / A \geq 7.0 \mathrm{MeV}$ is noticed. On the other hand, very neutron-rich nuclei with $N / Z \sim 2.0$ simultaneously start to be produced. By taking $E_{1}$ as the energy at which the peak height starts to be lowered (6.0 MeV in this case), and $E_{2}$ as the energy at which the yield of the equilibrated $N / Z$ becomes about $50 \%(7.0 \mathrm{MeV}$ in this case), the upper energy limit is defined in TDHF as $E_{\mathrm{CE}, \mathrm{cm}} / A=\left(E_{1}+E_{2}\right) / 2(6.5 \mathrm{MeV}$ in this case). The uncertainty from the energy bin value is $\left(E_{2}-E_{1}\right) / 2(0.5 \mathrm{MeV}$ in this case). Such TDHF results are summarized in the third and fourth columns of Table I. TDHF calculations with two different parameter sets result in the same upper energy limit. Note that a large value is

TABLE I. $\quad E_{\mathrm{CE}, \mathrm{cm}} / A$ values [MeV] obtained by TDHF calculations compared to those obtained by transforming the results of Eq. (1) into the center-of-mass frame. For reference, the values obtained by the Fermi gas model with the standard parameter are also shown.

\begin{tabular}{|c|c|c|c|c|c|}
\hline & Collision & TDHF (SLy4d) & $\mathrm{TDHF}\left(\mathrm{SkM}^{*}\right)$ & Equation (1) & Fermi gas \\
\hline (i) & ${ }^{208} \mathrm{~Pb}+{ }^{238} \mathrm{U}$ & $6.5 \pm 0.5$ & $6.5 \pm 0.5$ & 6.91 & 9.46 \\
\hline (ii) & ${ }^{208} \mathrm{~Pb}+{ }^{132} \mathrm{Xe}$ & $6.5 \pm 0.5$ & $6.5 \pm 0.5$ & 6.50 & 9.03 \\
\hline (iii) & ${ }^{208} \mathrm{~Pb}+{ }^{132} \mathrm{Sn}$ & $6.5 \pm 0.5$ & $6.5 \pm 0.5$ & 6.36 & 9.03 \\
\hline (iv) & ${ }^{208} \mathrm{~Pb}+{ }^{40} \mathrm{Ca}$ & $3.5 \pm 0.5$ & $3.5 \pm 0.5$ & 3.66 & 5.14 \\
\hline (v) & ${ }^{208} \mathrm{~Pb}+{ }^{24} \mathrm{Mg}$ & $2.5 \pm 0.5$ & $2.5 \pm 0.5$ & 2.36 & 3.52 \\
\hline (vi) & ${ }^{208} \mathrm{~Pb}+{ }^{24} \mathrm{O}$ & $2.5 \pm 0.5$ & $2.5 \pm 0.5$ & 2.18 & 3.52 \\
\hline (vii) & ${ }^{208} \mathrm{~Pb}+{ }^{16} \mathrm{O}$ & $1.5 \pm 0.5$ & $1.5 \pm 0.5$ & 1.75 & 2.50 \\
\hline (viii) & ${ }^{208} \mathrm{~Pb}+{ }^{4} \mathrm{He}$ & $<1.0$ & $<1.0$ & 0.48 & 0.70 \\
\hline (ix) & ${ }^{24} \mathrm{Mg}+{ }^{24} \mathrm{O}$ & $5.5 \pm 1.0$ & $5.5 \pm 1.0$ & 5.99 & 9.50 \\
\hline
\end{tabular}


obtained for ${ }^{24} \mathrm{Mg}+{ }^{24} \mathrm{O}$, implying that a simple mass dependence (small values for reactions between light nuclei, and vice versa) cannot explain this, while the mass asymmetry plays a certain role.

The corresponding values obtained by Eq. (1) are shown in the fifth column of Table I, and the values obtained by the simple Fermi gas model $\left(k_{F}=1.36 \mathrm{fm}^{-1}\right.$ for both protons and neutrons) are shown in the sixth column. By comparing the results of Eq. (1) with the TDHF results, the agreement is remarkable, including a high value in the last row. Figure 2 depicts how the upper limit given by Eq. (1) changes as a function of $N / Z$ and $A_{1} / A_{2}$. The upper energy limit comes down significantly low for higher mass asymmetry, while it depends only weakly on the total mass. Although charge equilibration can compete with Coulomb excitation particularly in collisions involving nuclei with large $Z$, no evidence of a major change due to large $Z$ is found in the results of Eq. (1) or those of TDHF calculations. However, non-negligible decrease of the upper energy limit due to the proton-neutron asymmetry is noticed. Consequently, the upper limit of charge equilibration depends largely on the Fermi energy, the proton-neutron asymmetry can contribute to the shift of the upper limit, and the dependence of the upper limit on the mass asymmetry is remarkable.

Let us move on to the comparison to the experiments. It can be seen that existing experimental data agree with the present upper-limit formula. For instance, the following experiments show charge equilibration: ${ }^{40} \mathrm{Ar}+{ }^{58} \mathrm{Ni}$ at $E_{\text {lab }} / A=7.0 \mathrm{MeV}$ [14], and ${ }^{56} \mathrm{Fe}+{ }^{165} \mathrm{Ho}\left({ }^{209} \mathrm{Bi}\right)$ at

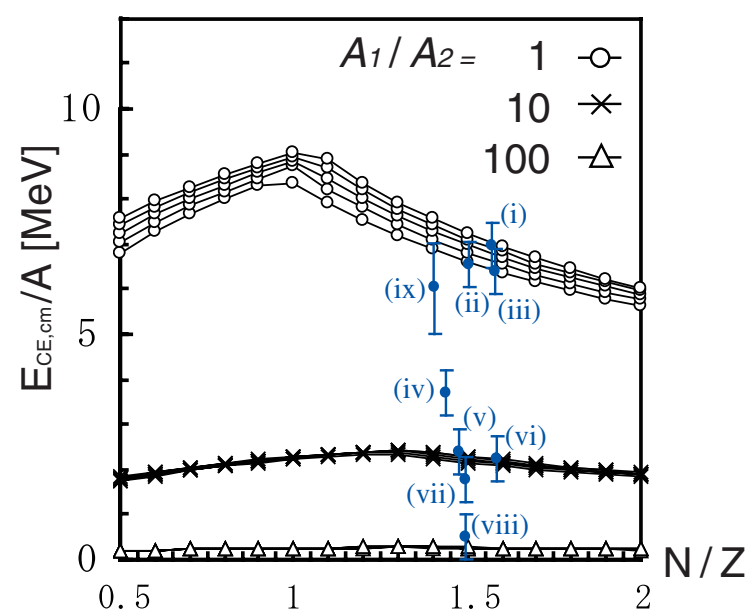

FIG. 2 (color online). $N / Z$ dependence of $E_{\mathrm{CE}, \mathrm{cm}} / A$ values [MeV] based on Eq. (1) in the center-of-mass frame, where $A_{1}>$ $A_{2}$ is assumed without loss of generality. Values with different total masses $A=100,200,300,400$, and 500 are plotted for each $A_{1} / A_{2}$, which correspond to black lines from bottom to top in each group (the total mass difference is not noticed for the cases of $A_{1} / A_{2}=10$ and 100), and the lines are drawn to guide eyes. Each TDHF calculation is shown as a blue bar, where the central points correspond to the value obtained by Eq. (1), and Roman numbers distinguish reactions shown in Table I.
$E_{\mathrm{lab}} / A=8.3 \mathrm{MeV}$ [15]. The following experiment shows the disappearance of charge equilibration: ${ }^{112} \mathrm{Sn}+{ }^{124} \mathrm{Sn}$ at $E_{\text {lab }} / A=50 \mathrm{MeV}[16,17]$. Recently, experiments ${ }^{124,112} \mathrm{Sn}+{ }^{124,112} \mathrm{Sn}$ at $E_{\mathrm{lab}} / A=35$ and $50 \mathrm{MeV}$, respectively, have been performed at Michigan State University [18]. It is remarkable that the final fragments are not so close to charge equilibrium even when the energy is set to $E_{\text {lab }} / A=35 \mathrm{MeV}$. This experimental result is understood now, because the upper limit of charge equilibration is calculated to be $E_{\text {lab }} / A=27.6 \mathrm{MeV}$ from Eq. (1).

The suppression of charge equilibration contributes naturally to the production of an exotic fragment; more exotic nuclei far from the equilibrated $N / Z$ ratio are to be synthesized above the present upper limit. Because the bombarding energies of the third generation RI-beam facilities are sufficiently high to exceed the upper limit, the present upper limit ensures more production of further exotic isotopes by the latest and the future RI-beam facilities. Indeed, in the experiment of Ref. [19], the yield of exotic fragments was increased simply by putting the beam energy higher than the present upper limit. It is also of much interest to explore the novel possibility of the synthesis of exotic nuclei by collisions including only $\beta$-stable nuclei. Such possibilities have not attracted much attention, but may emerge with various feasibilities of the production of exotic species, if the experiment is set for energies beyond the present upper limit. As an example, Fig. 3 shows the real-time dynamics of ${ }^{208} \mathrm{~Pb}+{ }^{40} \mathrm{Ca}$ around the energy where the production of exotic nuclei starts; the upper energy limit is $3.66 \mathrm{MeV}$ (Table I), fusion appears at $E_{\mathrm{cm}} / A=3.0 \mathrm{MeV}$, and breakup is seen at 4.0 MeV. $\beta$-unstable fragments are emitted only for the bombarding energy higher than the upper limit; for instance, a small fragment in the lower-right panel of Fig. 3 is ${ }^{26} \mathrm{Mg}$ (numbers of nucleon are rounded to be the integer).

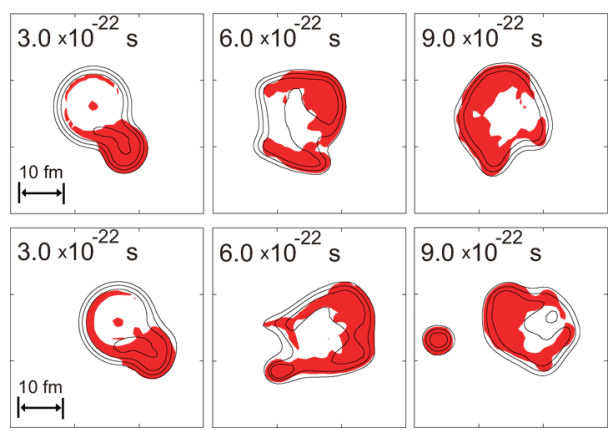

FIG. 3 (color online). Time evolution of charge distribution for ${ }^{208} \mathrm{~Pb}+{ }^{40} \mathrm{Ca}$ (SLy4d) is shown for the impact parameter of $7.5 \mathrm{fm}\left(b_{\mathrm{r}}=0.67\right)$. The upper and the lower panels show cases with $E_{\mathrm{cm}} / A=3.0 \mathrm{MeV}$ and $4.0 \mathrm{MeV}$, respectively. ${ }^{208} \mathrm{~Pb}$ is coming from the left for both cases. The contours incremented by $0.04 \mathrm{fm}^{-3}$ show the density, where parts with the protonneutron density ratio greater than 0.72 are colored in red. 


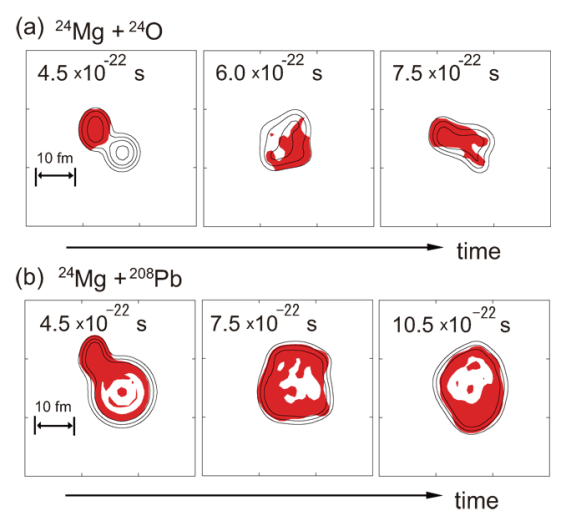

FIG. 4 (color online). Charge equilibration for (a) ${ }^{24} \mathrm{Mg}+{ }^{24} \mathrm{O}$ and (b) ${ }^{24} \mathrm{Mg}+{ }^{208} \mathrm{~Pb}$ (SLy4d) are shown for $E_{\mathrm{cm}} / A=4.5 \mathrm{MeV}$ and $2.0 \mathrm{MeV}$ with the impact parameters of 5.0 and $7.5 \mathrm{fm}\left(b_{\mathrm{r}}=\right.$ 0.72 and 0.71 ), respectively. For both cases, ${ }^{24} \mathrm{Mg}$ is incoming from the left and the states evolve into fusion. The description manner is the same as Fig. 3, where parts with the protonneutron density ratio greater than 0.80 and 0.70 are colored in red for (a) and (b), respectively.

We now point out some interesting details of chargeequilibration dynamics for collisions below the present upper energy limit. We shall first investigate it with a focus on the isovector dipole mode in a collision between light nuclei. Figure 4(a) shows the time-evolution of charge distribution for ${ }^{24} \mathrm{Mg}+{ }^{24} \mathrm{O}$. We see the appearance of the isovector dipole oscillation, and the charge equilibration is synchronized with it. Similar results were obtained in [2-10]. This is, however, seen mainly for collisions between light nuclei, because the dipole oscillation becomes less visible in heavier cases. Next, we move on to collisions involving a heavier nucleus, e.g., ${ }^{24} \mathrm{Mg}+{ }^{208} \mathrm{~Pb}$ shown in Fig. 4(b). No significant dipole oscillation appears. Instead, a radially layered structure of the composite nucleus is formed at $7.5 \times 10^{-22} \mathrm{~s}$, in which a relatively neutron-rich core appears in the center, a proton-rich layer surrounds it, and a neutron-rich skin is in the surface. This seems to be due to the Coulomb repulsion; hence, the radial distribution of charge is formed. Here one can find an analogous situation of isovector monopole excitation, and a prominent role of radial flow in such low-energy collisions is understood. Similar layered structures are obtained in the TDHF calculations listed in Table I except for ${ }^{24} \mathrm{Mg}+{ }^{24} \mathrm{O}$.

Finally, let us comment on the diffusion towards charge equilibrium. The mechanism of fast charge equilibration presented in this Letter is valid at energies lower than the upper energy limit. The diffusion may contribute to the charge equilibration around the Fermi energy [20-23], while its effect is not fast enough for attaining the charge equilibrium within the initial stage. A similar energydependent equilibration mechanism has been reported in condensed matter physics [24-26], where the equilibration is fast for lower energies (temperatures), and quite slow for higher energies.
In this Letter the mechanism of fast charge equilibration has been presented, and its validity is examined by comparison to virtually all existing relevant experimental data. This concept has been further analyzed in terms of a threedimensional time-dependent density functional formalism. The upper energy limit of charge equilibration has a crucial impact on the nuclear synthesis, giving a sound motivation for the production of further exotic isotopes by the latest and the future RI-beam facilities.

This work was supported by Helmholtz Alliance HA216/EMMI, by JSPS Core-to-Core program EFES, and by grant-in-aid for Scientific Research (A) 20244022. Y. I. thanks the JSPS for financial support, and expresses his gratitude to Dr. C. Simenel. T. O. thanks Professor P. Bonche for long-term valuable collaboration.

[1] H. Freiesleben and J. V. Kratz, Phys. Rep. 106, 1 (1984).

[2] M. Berlanger et al., Z. Phys. A 291, 133 (1979).

[3] E.S. Hernandez, W.D. Myers, J. Randrup, and B. Remaud, Nucl. Phys. A361, 483 (1981).

[4] P. Bonche and N. Ngô, Phys. Lett. 105B, 17 (1981).

[5] E. Suraud, M. Pi, and P. Schuck, Nucl. Phys. A492, 294 (1989).

[6] C. Simenel, Ph. Chomaz, and G. de France, Phys. Rev. Lett. 86, 2971 (2001).

[7] C. Simenel, Ph. Chomaz, and G. de France, Phys. Rev. C 76, 024609 (2007).

[8] V. Baran, D. M. Brink, M. Colonna, and M. Di Toro, Phys. Rev. Lett. 87, 182501 (2001).

[9] V. Baran, M. Colonna, V. Greco, and M. Di Toro, Phys. Rep. 410, 335 (2005).

[10] V. Baran, C. Rizzo, M. Colonna, M. Di Toro, and D. Pierroutsakou, Phys. Rev. C 79, 021603(R) (2009).

[11] W.D. Myers and W. J. Swiatecki, Ann. Phys. (N.Y.) 55, 395 (1969).

[12] W. D. Myers, Phys. Lett. 30B, 451 (1969).

[13] M. T. Collins and J. J. Griffin, Nucl. Phys. A348, 63 (1980).

[14] B. Gatty, D. Guerreau, M. Lefort, X. Tarrago, and J. Galin, Nucl. Phys. A253, 511 (1975).

[15] H. Breuer et al., Phys. Rev. Lett. 43, 191 (1979).

[16] P. M. Milazzo et al., Phys. Rev. C 66, 021601(R) (2002).

[17] W. P. Tan et al., Phys. Rev. C 64, 051901(R) (2001).

[18] M. B. Tsang et al. (private communication).

[19] M. Mocko et al., Phys. Rev. C 76, 014609 (2007).

[20] L. Shi and P. Danielewicz, Phys. Rev. C 68, 064604 (2003).

[21] M. B. Tsang et al., Phys. Rev. Lett. 102, 122701 (2009).

[22] M. Colonna, M. Di Toro, G. Fabbri, and S. Maccarone, Phys. Rev. C 57, 1410 (1998).

[23] J. Rizzo et al., Nucl. Phys. A806, 79 (2008).

[24] L. D. Landau and Zh. Eksperim, Sov. Phys. JETP 5, 101 (1957).

[25] W. R. Abel, A.C. Anderson, and J.C. Wheatley, Phys. Rev. Lett. 17, 74 (1966).

[26] A. J. Leggett, Rev. Mod. Phys. 47, 331 (1975). 